\title{
Enteral feeding of intrauterine growth restriction preterm infants: theoretical risks and practical implications
}

\author{
Valentina Bozzetti, Paolo E. Tagliabue \\ Neonatal Intensive Care Unit, MBBM Foundation, San Gerardo Hospital, Monza, Italy
}

\begin{abstract}
Intrauterine growth restriction (IUGR) infants are thought to have impaired gut function after birth secondary to intrauterine redistribution of the blood flow, due to placental insufficiency, with a consequent reduction of gut perfusion. For this reason, infants complicated by IUGR have been considered at higher risk of feeding intolerance. Postnatal evaluation of splanchnic perfusion, through Doppler of the superior mesenteric artery, and of splanchnic oxygenation, through near infrared spectroscopy measurements, may be useful in evaluating the persistence (or not) of the redistribution of blood flow occurred in utero.
\end{abstract}

\section{Introduction}

The term intrauterine growth restriction (IUGR) indicates the presence of a pathological process occurring in utero that inhibits fetal growth as documented by at least two fetal intra-uterine growth assessments. Fetal growth restriction (FGR) or intrauterine growth restriction is defined as the failure of the fetus to achieve its growth potential and represents one of the most debated issues in obstetrics, being in continuous search for improvement in terms of definition, classification, diagnosis and management. ${ }^{1}$ IUGR is an important problem in perinatal medicine. It is the second cause of perinatal mortality after prematurity. The two conditions are

Correspondence: Valentina Bozzetti, Neonatal Intensive Care Unit, MBBM Foundation, San Gerardo Hospital, via Pergolesi 33, 20900 Monza, Italy.

Email: vbozzetti@hotmail.com

Key words: Enteral feeding; IUGR; Preterm infants; Risks.

Funding: Funded by Merqurio Editore S.r.1., with the unconditional contribution of Nestlè Italiana S.p.A.

Received for publication: 7 June 2017.

Accepted for publication: 21 June 2017

This work is licensed under a Creative Commons Attribution

NonCommercial 4.0 License (CC BY-NC 4.0).

CC Copyright V. Bozzetti and P.E. Tagliabue, 2017

Licensee PAGEPress, Italy

La Pediatria Medica e Chirurgica 2017; 39:160

doi:10.4081/pmc.2017.160 often associated in case of iatrogenic prematurity. Fifty-two per cent of stillbirths are associated with IUGR and $10 \%$ of perinatal mortality cases in Europe are the consequence of unrecognized severe growth restriction. In developed countries, 3-7\% of newborns are classified as IUGR. ${ }^{2}$

The most common cause of IUGR is the placental obliterative vasculopathy that reduces nutrients and oxygen supply to the fetus; the causes include, but are not limited to, maternal malnutrition, maternal hypertension and idiopathic placental insufficiency. Placental insufficiency may be characterized by blood flow redistribution to the vital organs (brain, myocardium, and adrenal glands), while other organs, including the gastrointestinal tract, are deprived from sufficient blood flow. These fetuses are at increased risk of hypoxia, hypoglycemia and acidosis and also spontaneous preterm delivery. 3,4

\section{The intrauterine growth restriction fetus: detection and monitoring}

\section{Fetal monitoring}

The main directions towards assessing fetal growth state can be clinical (e.g. the assessment of symphyseal-fundal height) and paraclinical (e.g. ultrasound biometry).

Commonly used clinical methods for estimating fetal size are clinical palpation, fundal height measurement and ultrasonic fetal biometry. Serial ultrasound biometric recordings may be able to identify the fetus that does not reach its growth potential. Ultrasound must be considered the method of choice as it is highly reliable and reproducible. 5 The ultrasound limit for SGA or FGR is arbitrarily fixed at an estimated fetal weight (EFW) or abdominal circumference $(\mathrm{AC})$ under the $3^{\text {rd }}, 5^{\text {th }}, 10^{\text {th }}$ percentile or below -2 Standard Deviation (SD) from the population standard or reference. 6

When IUGR is the consequence of a placental etiology (placental insufficiency), management is based on the careful fetal assessment to detect the optimal time for delivery. The most commonly used methods of monitoring include Doppler assessment. Doppler velocity waveform in arteries is mainly influenced by the characteristics of the diastolic phase and reflects the peripheral resistance to blood flow. The pulsatility index (PI) (peak systolic velocity - end-diastolic velocity/mean velocity) is commonly used. PI values increase as the peripheral resistance increases. ${ }^{1}$

Increased placental resistance in the presence of placental failure leads to a reduction in end diastolic blood flow (EDF) through the umbilical arteries (UA), progressing to absent (AEDF) or reversed flow (AREDF). ${ }^{7}$ Animal and human experiments have shown that there is an increase in blood flow to the brain in the 
IUGR fetus. This increase in blood flow can be evidenced by Doppler ultrasound of the middle cerebral artery (MCA). Longitudinal studies on deteriorating early-onset IUGR fetuses have reported that MCA PI progressively becomes abnormal. This phenomenon is defined as brain sparing (BS) effect. An UA PI to MCA PI ratio $>1$ is considered as index of BS and fetal hypoxia. 8 Up to $20 \%$ of IUGR fetuses have severe Doppler abnormalities also associated with poorer perinatal outcome and suboptimal neurodevelopmental development at 2 years of age.9-11 As a consequence of the redistribution of the blood flow in IUGR infants, gut may be under-perfused and ischemia/hypoxia may occur; IUGR infants are thought to have impaired gut function after birth, which may result in intestinal disturbances, ranging from temporary intolerance to the enteral feeding to full-blown necrotizing enterocolitis (NEC) occurring up to $7 \%$ of very low birth weight infants. ${ }^{12-15} \mathrm{~A}$ meta-analysis of 14 observational studies demonstrated an increased incidence of NEC in preterm infants who had suffered fetal AREDF compared with controls, with an odds ratio of 2.13 ( $95 \%$ CI 1.49 to 3.03$) .16$

\section{Neonatal monitoring Doppler findings}

The superior mesenteric artery (SMA) is the major source of blood for the small intestine and for a portion of the large intestine. After birth, SMA blood flow velocity (BFV) increases to support the dramatic boost to in intestinal growth and oxygen uptake that occurs during the first few postnatal weeks. ${ }^{17}$ There is increasing evidence that the rate of increase in SMA BFV may have clinical significance. Greater increases in postnatal SMA BFV during the first week of life in preterm infants are reported to be associated with less intestinal dysmotility, and with better tolerance to enteral feedings. ${ }^{18,19}$ Intestinal blood flow is regulated by numerous factors including cardiovascular status, neural control, humoral substances and local control. Extrinsic factors that can affect splanchnic blood flow in preterm infants include the type, modality and volume of enteral nutrition given, and various pharmacologic agents, including indomethacin and caffeine. Although factors that can affect intestinal blood flow have been identified, little is known about factors that may affect the rate at which SMA BFV increases in newborn preterm infants. ${ }^{20-23}$

\section{Near infrared spectroscopy}

The assessment of adequate perfusion in very low birth weight infants is commonly based on clinical parameters, as well as invasive measures requiring central venous and/or arterial catheter access, associated with well-known risks. Since the pathogenesis of intestinal dysfunction in IUGR infants seems to be related with a redistribution of the blood flow, monitoring the perfusion and the oxygenation of the splanchnic district in IUGR infants is mandatory. Furthermore, most of these data are acquired intermittently, and thus may only represent a delayed picture of oxygen delivery and consumption.

Near infrared spectroscopy (NIRS) is a continuous, non-invasive, real-time and portable technique, which can be used to measure oxygenation in living tissue. ${ }^{24}$ NIRS has been reported to be useful in detecting changes in oxygen delivery. Thus, NIRS in the splanchnic district offers useful information on tissue oxygen delivery and may predict splanchnic ischemia in neonates by measuring, the cerebrosplanchnic oxygenation ratio (CSOR). ${ }^{25}$ NIRS can detect changes in splanchnic oxygen delivery, which is curtailed during IUGR and may be used to predict feeding intolerance and NEC by measuring the splanchnic/cerebral oxygenation status ratio.

\section{Intrauterine growth restriction and preterm infants feeding: risks and practical implications}

Intrauterine hypoxia of the gut and increased oxygen demand during enteral feeds in a vulnerable and immature host are associated with feeding difficulties and increased risk of necrotising enterocolitis (NEC) in these neonates. For this reason, infants born prematurely and IUGR, especially those with extremely low birth weight (ELBW), are often considered to be too unstable to be fed enterally. Nevertheless, the use of a prolonged parenteral nutrition (PN) exposes infants to the metabolic and central line complications as well as to the deleterious effects of fasting on gastrointestinal system.26,27 Late introduction of feeding may be detrimental due to lack of stimulation of the gastrointestinal tract, resulting in villous atrophy and lack of hormone and enzyme production and may not reduce the incidence of NEC. Enteral fasting can also affect the timing of full enteral feeding (FEF) and may delay hospital discharge. 28,29

MEF is also known as trophic feeding or gut-priming or non nutritive feeding or hypocaloric feeding conventionally defined as giving small volumes of milk (typically 12 to $24 \mathrm{~mL} / \mathrm{kg} /$ day) starting within the first few days after birth without advancing the feed volumes during the first week of life. ${ }^{30} \mathrm{MEF}$ in combination with PN are employed to: i) promote the intestinal motility and the development of an appropriate microflora, ii) preserve intestinal barrier integrity, and iii) reduce infection's rate.

Early enteral feeding is advantageous because it improves the functional adaptation of the gastrointestinal tract by stimulating hormone secretion and gastrointestinal motility. ${ }^{31}$ MEF has also been shown to improve gastrointestinal disaccharidase activity, hormone release, blood flow, motility and microbial flora. Clinical benefits include improved milk tolerance, greater postnatal growth, reduced systemic sepsis and shorter hospital stay.

A meta-analysis of RCTs conducted on preterm infants did not detect a significantly different risk of NEC between infants randomized to delayed feeding (as later than day 5-7 after birth) and infants $v$ s early feeding (less than 4 day after birth). ${ }^{32-35}$

An RCT was conducted to assess tolerance of feeding in preterm infants with IUGR. Infants with gestation below 35 weeks, birth weight below the 10th centile, and abnormal antenatal umbilical artery Doppler waveforms were randomly allocated to commence enteral feeds early, on day 2 after birth, or late, on day 6 . Gradual increase in feeds was guided by a feeding prescription with rate of increase the same for both groups. Primary outcomes were time to achieve full enteral feeding sustained for 72 hours and NEC. Early introduction of enteral feeds in growth-restricted preterm infants resulted in earlier achievement of full enteral feeding and did not appear to increase the risk of NEC. ${ }^{36}$

Kempley et al. ${ }^{37}$ have recently conducted a post-hoc analysis of subgroup data from the cited above ADEPT trial. They reported that growth-restricted neonates with a gestational age (GA) below 29 weeks gestation with abnormal antenatal Doppler failed to tolerate even the careful feeding. Full feeds were achieved later in neonates with GA $<29$ weeks compared with GA $\geq 29$ weeks. Neonates $<29$ weeks tolerated very little milk for the first 10 days of life and reached full feeds 9 days later than predicted from the trial regimen. Thus, it is strongly recommended to be extremely cautious in advancing enteral feeding in IUGR extremely preterm infants.

Accordingly, a retrospective analysis performed in our NICU provided evidence that feeding tolerance improves as gestational age increases and impairs in small for gestational age infants. Furthermore, SGA infants in our cohort spent more time to achieve 
full enteral feeding than AGA infants. This could be a consequence of gut ischemia/hypoxia, due to placentar insufficiency, with consequent impaired gut function after birth, which may result in feeding intolerance. 38

When IUGR is complicated by brain sparing (BS) effect, a redistribution of cardiac output to maintain oxygen supply to the brain, heart, and adrenal glands, the gastrointestinal tract may be deprived by adequate blood flow. Therefore, infants complicated by BS are thought to be at major risk of feeding intolerance. To assess if the prenatal monitoring parameters, such as prenatal Doppler' patterns, directly impact on tolerance of enteral nutrition we performed a case-control study investigating whether in IUGR infants the occurrence or not of brain sparing effect may interfere with the timing necessary to achieve full enteral feeding. We found that achievement of full enteral feeding was influenced by abnormal Doppler patterns suggestive of fetal hypoxia. ${ }^{39}$

Although hemodynamic patterns related to BS have been widely described, 40,41 data on perinatal adaptation in IUGR infants and the timing of the exhaustion of the BS effect are still matters of investigation. Data for the BS effect on splanchnic perfusion and oxygenation status after birth and in the transitional period were investigated by using NIRS in a prospective study on preterm IUGR infants. Results of this research provide evidence that in IUGR infants the pattern of regional cerebral and splanchnic oxygen status, suggestive of a brain sparing effect, is still present in the transition phase and differs from controls as suggested by the cerebral/splanchnic ratio. ${ }^{42} \mathrm{In}$ an RCT conducted in our Department we analyzed whether the effect exerted by feeding on intestinal perfusion, evaluated with Doppler of superior mesenteric artery (SMA), was different among preterm infants with or without IUGR. In our study, IUGR and NON IUGR infants showed a similar increase in blood-flow velocity in SMA after feedings. These findings suggest that IUGR infants, who are unable to develop the physiological postprandial increase of BFV in the SMA after the first feeding in the first days of life, ${ }^{43}$ may acquire this ability later on, when they tolerate full orogastric feeds. ${ }^{44}$

\section{Conclusions}

Altogether there is evidence that feeding issue in preterm and IUGR infants still constitutes a hot topic in NICU daily practice opening-up to further studies in terms of: i) how and when to start feeding in IUGR and preterm infants, and ii) usefulness of perinatal standard monitoring procedures for feeding start.

\section{References}

1. Ghidini A. Idiopathic fetal growth restriction: a pathophysiologic approach. Obstet Gynecol Surv 1996;51:376-82.

2. Mandruzzato G. Intrauterine growth restriction (IUGR). J Perinat Med 2008;36:277-81.

3. Lackman F, Capewell V, Gagnon R, Richardson B. Fetal umbilical cord oxygen values and birth to placental weight ratio in relation to size at birth. Am J Obstet Gynecol 2001; 185:674-82

4. Economides DL, Nicolaides KH, Campbell S. Relation between maternal-to-fetal blood glucose gradient and uterine and umbilical Doppler blood flow measurements. Brit J Obstet Gynaecol 1990;97:543-4.

5. Perni CS, Chervenak FA, Kalish RB, et al. Intraobserver and interobserver reproducibility of fetal biometry. Ultrasound Obstet Gynecol 2004;24:654-8.

6. Unterscheider J. Optimizing the definition of intrauterine growth restriction: the multicenter prospective PORTO Study. Am J Obstet Gynecol 2013;208:1-6.

7. Baschat AA. Fetal responses to placental insufficiency: an update. BJOG 2004;111:1031-41.

8. Eronen M, Kari A, Pesonen E, et al. Value of absent or retrograde end-diastolic flow in fetal aorta and umbilical artery as a predictor of perinatal outcome in pregnancy-induced hypertension. Acta Paediatr 1993;82:919-24.

9. Gilbert WM, Danielsen B. Pregnancy outcomes associated with intrauterine growth restriction. Am J Obstet Gynecol 2003;188:1596-9.

10. Aucott SW, Donohue PK, Northington FJ. Increased morbidity in severe early intrauterine growth restriction. J Perinatol 2004;24:435-40.

11. Eixarch E, Meler E, Iraola A, et al. Neurodevelopmental outcome in 2-year-old infants who were small-for-gestational age term fetuses with cerebral blood flow redistribution. Ultrasound Obstet Gynecol 2008;32:894-9.

12. Baserga M, Bertolotto C, Maclennan NK, et al. Uteroplacental insufficiency decreases small intestine growth and alters apoptotic homeostasis in term intrauterine growth retarded rats. Early Hum Dev 2004;79:93-105.

13. Xu RJ, Mellor DJ, Birtles MJ, et al. Impact of intrauterine growth retardation on the gastrointestinal tract and the pancreas in newborn pigs. J Pediatr Gastroenterol Nutr 1994;18: 231-40.

14. Baschat AA, Hecher K. Fetal growth restriction due to placental disease. Semin Perinatol 2004;28:67-80.

15. Berman L, Moss RL. Necrotizing enterocolitis: an update. Semin Fetal Neonatal Med 2011;16:145-50.

16. Dorling J, Kempley S, Leaf A. Feeding growth restricted preterm infants with abnormal antenatal Doppler results. Arch Dis Child Fetal Neonatal Ed 2005;90:359-63.

17. Reber KM, Nankervis CA, Nowicki PT. Newborn intestinal circulation. Physiology and pathophysiology. Clin Perinatol 2002;29:23-39.

18. Robel-Tillig E, Knupfer M, Pulzer F, Vogtmann C. Blood flow parameters of the superior mesenteric artery as an early predictor of intestinal dysmotility in preterm infants. Pediatr Radiol 2004;34:958-62.

19. Maruyama K, Koizumi T, Tomomasa T, Morikawa A. Intestinal blood-flow velocity in uncomplicated preterm infants during the early neonatal period. Pediatr Radiol 1999; 29:472-7

20. Carver JD, Saste M, Sosa R, et al. The effects of dietary nucleotides on intestinal blood flow in preterm infants. Pediatr Res 2002;52:425-9.

21. Leidig E. Doppler analysis of superior mesenteric artery blood flow in preterm infants. Arch Dis Child 1989;64:476-80.

22. Martinussen M, Brubakk AM, Vik T, Yao AC. Mesenteric blood flow velocity and its relation to transitional circulatory adaptation in appropriate for gestational age preterm infants. Pediatr Res 1996;39:275-80.

23. Yanowitz TD, Yao AC, Pettigrew KD, et al. Postnatal hemodynamic changes in very-low-birthweight infants. J Appl Physiol 1999;87:370-80

24. Hoffman GM, Stuth EA, Berens RJ, et al. Two-site nearinfrared transcutaneous oximetry as a non-invasive indicator of mixed venous oxygen saturation in cardiac neonates. Anesthesiology 2003;98:A1393.

25. Fortune PM, Wagstaff M, Petros AJ. Cerebro-splanchnic oxy- 
genation ratio (CSOR) using near infrared spectroscopy may be able to predict splanchnic ischaemia in neonates. Intensive Care Med 2001;27:1401-7.

26. Camara D. Minimizing risks associated with peripherally inserted central catheters in the NICU. MCN Am J Matern Child Nurs 2001;26:17-21.

27. Schutzman DL, Porat R, Salvador A, Janeczko M. Neonatal nutrition: a brief review. World J Pediatr 2008;4:248-53.

28. Lucas A, Bloom SR, Aynsley-Green A. Gut hormones and 'minimal enteral feeding'. Acta Paediatr Scand 1986;75: 719-23.

29. Berseth CL. Neonatal small intestinal motility: motor responses to feeding in term and preterm infants. J Pediatr 1990, 117:777-82.

30. McClure RJ: Trophic feeding of the preterm infant. Acta Paediatr Supp 2001;90:19-21.

31. Burrin DG, Stoll B. Key nutrients and growth factors for the neonatal gastrointestinal tract. Clin Perinatol 2002;9:65-96.

32. Morgan J, Young L, McGuire W. Delayed introduction of progressive enteral feeds to prevent necrotising enterocolitis in very low birth weight infants. Cochrane Database Syst Rev 2011;3:CD001970.

33. Karagianni P, Briana DD, Mitsiakos G, et al. Early versus delayed minimal enteral feeding and risk for necrotizing enterocolitis in preterm growth-restricted infants with abnormal antenatal Doppler results. Am J Perinatol 2010;27:367-73.

34. Leaf A, Dorling J, Kempley S, et al. Early or delayed enteral feeding for preterm growth-restricted infants: a randomized trial. Pediatrics 2012;129:e1260-8.

35. Van Elburg RM, van den Berg A, Bunkers CM, et al. Minimal enteral feeding, fetal blood flow pulsatility, and postnatal intes- tinal permeability in preterm infants with intrauterine growth retardation. Arch Dis Child Fetal Neonatal Ed 2004;89:293-6.

36. Leaf A, Dorling J, Kempley S, et al. Early or delayed enteral feeding for preterm growth-restricted infants: a randomized trial. Pediatrics 2012;129:e1260-8.

37. Kempley S, Gupta N, Linsell L, et al. Feeding infants below 29 weeks' gestation with abnormal antenatal Doppler: analysis from a randomised trial. Arch Dis Child Fetal Neonatal Ed 2014;99:6-11.

38. Bozzetti V, Tagliabue PE, Visser GH, et al. Feeding issues in IUGR preterm infants. Early Hum Dev 2013;89(Suppl.2):21-3.

39. Bozzetti V, Paterlini G, Gazzolo D, et al. Monitoring Doppler patterns and clinical parameters may predict feeding tolerance in intrauterine growth-restricted infants. Acta Paediatr 2013;102:e519-23.

40. Westergaard HB, Langhoff-Roos J, Lingman G, et al. A critical appraisal of the use of umbilical artery doppler ultrasound in high-risk pregnancies: use of meta-analyses in evidence-based obstetrics. Ultrasound Obstet Gynecol 2001;17:466-76.

41. Hasegawa J, Nakamura M, Matsuoka R, et al. Evaluation of placental function using near infrared spectroscopy during fetal growth restriction. J Perinat Med 2010;38:29-32.

42. Bozzetti V, Paterlini G, Bel Fv, et al. Cerebral and somatic NIRS-determined oxygenation in IUGR preterm infants during transition. J Matern Fetal Neonatal Med 2016;29:443-6.

43. Maruyama K, Fujiu T, Inoue T, et al. Feeding interval and postprandial intestinal blood flow in premature infants. Pediatr Int 2013;55:472-6.

44. Bozzetti V, Paterlini G, De Lorenzo P, et al. Impact of continuous vs bolus feeding on splanchnic perfusion in very low birth weight infants: a randomized trial. J Pediatr 2016;176:86-92. 